\title{
Comparing Two Different Concentrations of Diphenhydramine Versus Lidocaine for Median Nerve Block in Emergency Department: A Double-Blind Randomized Clinical Trial
}

\author{
(1) Shervin Farahmand ${ }^{1}$, (1) Fariba Yazdanbakhsh ${ }^{1}$, (1) Shahram Bagheri-Hariri¹, (1) Ehsan Aliniagerdroudbari², \\ (1) Atefeh Abdollahi ${ }^{1}$, (1) Sepideh Babaniamansour ${ }^{3}$
}

1Prehospital and Hospital Emergency Research Center, Tehran University of Medical Sciences, Tehran, Iran

2School of Medicine, Shahid Beheshti University of Medical Sciences, Tehran, Iran

${ }^{3}$ School of Medicine, Islamic Azad University of Tehran Medical Sciences, Tehran, Iran

\begin{abstract}
Aim: This study aimed to compare two different concentrations of Diphenhydramine vs. Lidocaine for median nerve block in terms of providing required anesthesia for repairing soft tissue injuries.

Materials and Methods: This double-blind, randomized clinical trial was conducted in Tehran, Iran, in 2019. Participants were randomly assigned to three groups. Group A received 3 cc of $0.5 \%$ diphenhydramine $(n=68)$, group B received 3 cc of $1 \%$ lidocaine ( $n=68$ ), and group C received 3 cc of $1 \%$ diphenhydramine $(n=68)$. The onset of action, duration of anesthesia, pain during injection, and patients' satisfaction level were evaluated between the groups.

Results: Two hundred two patients with the mean age of $35.09 \pm 13.5$ years took part in this study ( $52.5 \%$ of the males). Age ( $p=0.879)$, onset of action $(p=0.251)$ and duration of anesthesia $(p=0.081)$ had no significant difference among the three groups. The pain during injection between groups A and B $(p=0.001)$ and between groups B and C $(p<0.0001)$ had significant differences. Patients in group B had the significant highest level of satisfaction $(p<0.0001)$.

Conclusion: In our study, two different concentrations of diphenhydramine had the same onset of action and duration of anesthesia compared to lidocaine. Lidocaine caused lower pain during injection compared to diphenhydramine, and the level of satisfaction was higher with lidocaine. Diphenhydramine had no significant difference with different concentrations in terms of pain during injection.
\end{abstract}

Keywords: Hospital emergency service, diphenhydramine, lidocaine, pain measurement, median nerve

\section{Introduction}

The use of local nerve anesthesia for hands is becoming increasingly common in emergency procedures. Emergency medicine physicians (EMPs) are conducting many simple intradermal procedures on the hand, e.g. laceration repair. A local anesthetic is an integral aspect of the procedures which consists of the specific structure for penetrating the lipid rich nerve membrane and water solubilizing and chain for making this connection. It blocks the autonomic, sensory and motor nervous impulses by preventing the sodium influx to the cells and membrane depolarization (1-3). The local anesthesia is mostly classified in to esters group such as procaine and tetracaine and amides group such as lidocaine and bupivacaine that have both negative and positive points (4). Therefore, choosing the best anesthetic agent to control pain with the highest efficacy and least pain at the site of injection is of paramount importance. EMPs are always looking for an anesthetic agent with an appropriate 
onset time and duration that causes the least adverse effects. Lidocaine is the safest and the most popular anesthetic drugs in the emergency department (ED) and is used in many procedures due to its short onset action. However, some patients reported an unclear or true history of hypersensitivity which makes the choice of the best anesthetic agent difficult (2,5-7). In many studies, diphenhydramine was selected as an alternative local anesthetic in such situations (6). The main use of diphenhydramine is as a first-generation antihistamine, but it also acts as a local anesthetic because of its three-dimensional structure like an amine terminus that is similar to other anesthetic drugs $(7,8)$. A study in 2009 showed that $80 \%$ of patients realized the onset of anesthetization within five minutes following injection of a $1 \%$ solution of diphenhydramine, and that anesthetization lasted 15 minutes to three hours. However, the pain related to the injection of diphenhydramine was higher than that of lidocaine (7). Although some studies found pain and irritation from diphenhydramine injection, most studies did not show any allergic cross-reactivity (8).

Given the limited number of studies comparing the efficacy of diphenhydramine in different doses as a neural blocking agent especially for median nerve block.

This study aimed to compare two different concentrations of Diphenhydramine vs. Lidocaine for median nerve block in terms of providing required anesthesia for repairing soft tissue injuries.

\section{Materials and Methods}

\section{Trial Design}

This study was a double-blind clinical trial conducted at Imam Khomeini Hospital Complex affiliated to Tehran University of Medical Science, Tehran, Iran,during 2019. The executive protocol of the study was approved by the Ethics Committee of Tehran University of Medical Sciences (ethic code: IR.TUMS.IKHC. REC.1397.095) and registered in the Iranian Registry of Clinical Trials (registration number: IRCT20190318043088N1). The study was conducted in accordance with the Declaration of Helsinki Principles. Written informed consent was taken from all patients prior to their participation in the study. This research did not receive any specific grant from funding agencies in the public, commercial, or not-for-profit sectors.

\section{Participants}

Participants in the study were patients referred to the ED between the age of 16 and 65 years with an indication for Median nerve block (including lacerations to the palmar surface of hand, thumb, second or third finger or radial half of fourth finger or the nailbed of these fingers or the thenar eminence and lumbrical muscles of the first and second finger). Patients excluded from this study were those who had known allergy to diphenhydramine or lidocaine, those who received any systemic anesthesia prior to the intervention and those who received monoamine oxidase (MAO) inhibitors (due to prolongation of MAO inhibitors anticholinergic effects) or barbiturates (due to increasing central nervous system sedation). Breastfeeding mothers, patients with severe asthma, uncontrolled hypertension, glaucoma, ischemic heart diseases, benign prostatic hyperplasia, hyperthyroidism, or pyloroduodenal obstruction were also excluded.

\section{Interventions}

Group A received 3 cc of $0.5 \%$ diphenhydramine, group B received 3 cc of $1 \%$ lidocaine, and group C received 3 cc of $1 \%$ diphenhydramine (9-11). The Caspian Tamin Pharmaceutical Co (Tehran, Iran) was the manufacturer of medications. Using 0.05 inch 27-gauge needles, median nerve block was performed by injecting $3 \mathrm{~mL}$ of the local anesthetic solution medial to the flexor carpi radialis tendon, 2 to 3 proximal to the wrist crease on a flat surface after cleaning area using chlorhexidine $2 \%$. The solution was injected at a 45-degree angle to a depth of $16 \mathrm{~mm}$ (12). The anesthetization in all three groups of patients was assessed at 5, 10- and 15-minute intervals following administration of the anesthesia. The procedure ended whenever the patient declared they were pain free. Patients who were not anesthetized after 15 minutes, or who expressed dissatisfaction with the result, would be administered by $1 \%$ Lidocaine as a rescue dose.

\section{Outcomes}

The primary outcome including the pain score at injection site, onset of action (defined as the time when pain started to decrease) and duration of anesthesia (defined as the time from the onset action to the relapse of sensation) were assessed. At the end of the procedure, the patient's satisfaction with pain control during the procedure and the adverse effects during procedure or later (before ED discharge) were recorded as secondary outcomes. The satisfaction was recorded using a five-point Likert scale (not satisfied, slightly satisfied, satisfied, very satisfied, and extremely satisfied). The variables were compared among different treatment groups and between genders.

\section{Sample Size}

The sample size was calculated using the formula below and considering $\alpha=0.05$ and power of $80 \%$ as at least 14 in each arm of the study.

$$
\begin{aligned}
& N=\frac{\left(Z_{1 \alpha_{/ 2}}+Z_{\beta}\right)^{2}\left[\left(S D_{1}\right)^{2}+\left(S D_{2}\right)^{2}\right]}{\left(M_{1}-M_{2}\right)^{2}} \\
& \begin{array}{l}
\alpha=0.05 \rightarrow Z_{1-\alpha / 2}=1.96 \\
\beta=0.2 \rightarrow Z_{\beta}=0.84
\end{array}
\end{aligned}
$$


Diphenhydramine: $\mathrm{M} 1=1.56$ and SD1 $=0.54$, Lidocaine: $\mathrm{M} 2=2.06$ and SD2 $=0.41$

\section{Primary Assessment}

Assessment of the patient's pain score was based on the Visual Analog Scale (VAS) a psychometric scale used to indicate the pain intensity at an injection site. Patients mark their level of pain along a straight-line scale from zero to 10 where patients without pain get zero and patients with the highest intensity of pain get $10(13,14)$.

\section{Randomization and Blinding}

Randomization was done using computer-generated allocation table provided by RANDOM.ORG website (15), encoded with letter A, B or C by the supervisor of the project. Color and appearance of the drugs were identical and administered by the same-size syringes, percutaneously. A nurse was responsible for administration of drugs. Patients, nurse and statistical analyst were all blinded to their assessments.

\section{Statistical Analysis}

SPSS Version 22.0 (SPSS Inc., Chicago, IL, USA) was used to analyze the data. Quantitative variables were described using the mean \pm SD and qualitative variables were described using the frequency and percentage of the data. Three variables were measured during the procedures: the pain score reported by the patients during the injection, the length of time to onset action, and the duration of anesthesia. The relationship between these three variables among the treatment groups was examined using One-way ANOVA and Tukey test. The relationship of onset action, duration of anesthesia and pain score between the genders was examined using independent sample t-test. The relation between categorial variables was examined using chi-square test and Fisher's exact test. $\mathrm{P}<0.05$ was considered statistically significant.

\section{Results}

Two hundred four patients took part in the study (68 in each group). Figure 1 shows the CONSORT flowchart of the study patients. One patient from group B and one from group C reported a relapse of pain, 20 minutes after the injection. Those two patients required rescue intervention for injecting to the border of laceration and excluded from the analysis.

Finally, the data from 202 patients were analyzed of whom 52.5\% were male. The mean age was $35.09 \pm 13.5$ years (maximum and minimum of 16 and 64$)$. The satisfaction level $(p=0.662)$, onset action $(p=0.667)$, duration of anesthesia $(p=0.832)$ and

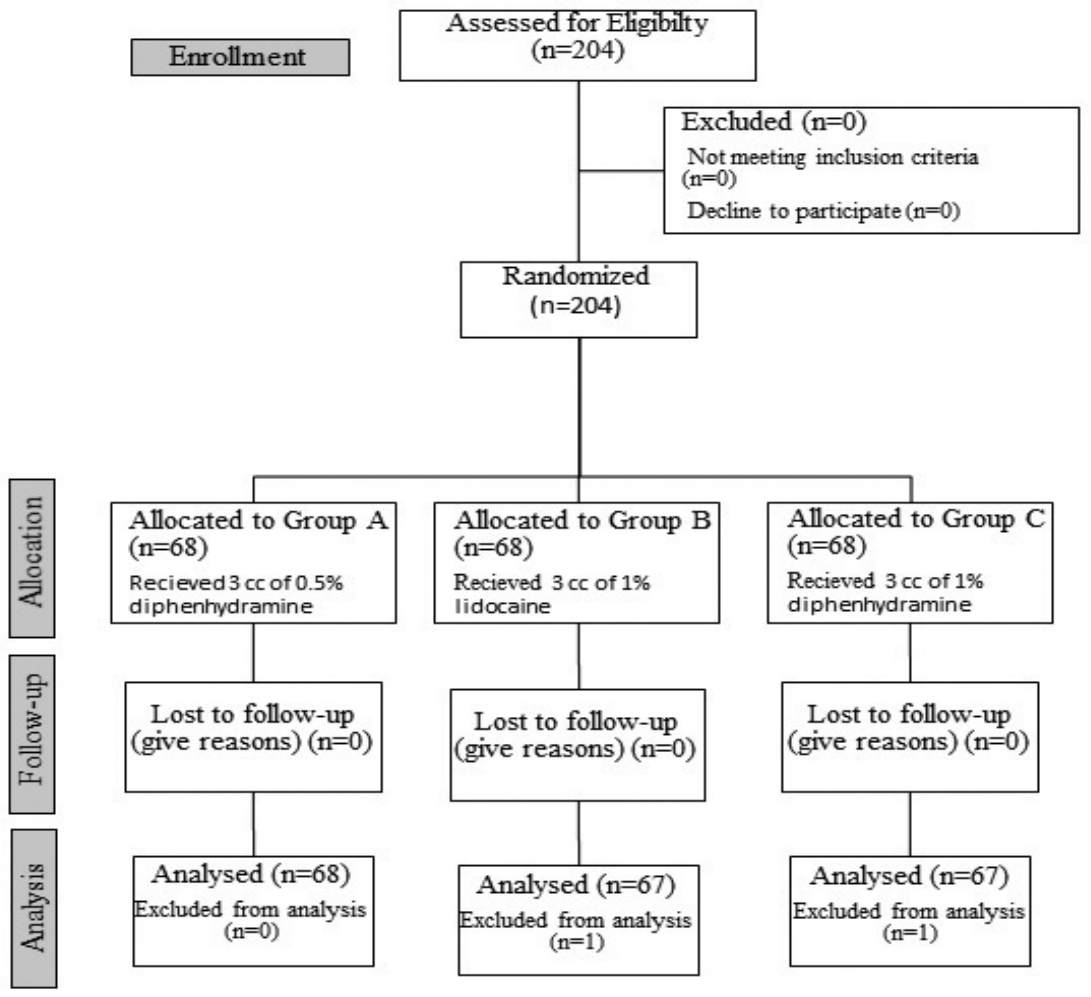

Figure 1. CONSORT flow diagram of the trial: CONSORT flow diagram showing participant flow through each stage of the randomized controlled trial (enrolment, intervention allocation, follow-up and data analysis)

CONSORT: Consolidated Standards of Reporting Trials, n: Number 
pain score $(p=0.179)$ had no significant difference between the genders.

Comparing the primary outcomes among treatment groups showed that onset action, duration of anesthesia and pain score during injection were lower in group B, A and B, respectively. Comparing the secondary outcomes showed that patients in group A and C mostly reported "slightly satisfied" and patients in group B mostly reported "very satisfied". In addition, the frequency of adverse effect (erythema, drowsiness and both) was found only in $4.46 \%$ of patients which was higher in group C. (Table 1). Age $(p=0.879)$, onset action $(p=0.251)$ and duration of anesthesia $(p=0.081)$ had no significant difference among treatment groups. There was a significant difference in reported pain score during injection among groups $(p<0.0001)$. Tukey test (HSD) showed that the difference in pain during injection between groups $A$ and $B(p=0.001)$ and between groups $C$ and $B(p<0.0001)$ were statistically significant, but there was no significant difference between groups $A$ and $C,(p=0.367)$. The confidence interval of the pain score during injection ranged from 4.5 to 5.3 in group A, from 3.6 to 4.3 in group B and from 5 to 5.7 in group C. (Figure 2) The Tukey test put groups A and $C$ with similar mean pain score during injection into a cluster and group B in another cluster. There was significant difference between patient's satisfaction level of different treatment groups $(p<0.0001)$.

\section{Discussion}

This study compared the effects of two different doses of diphenhydramine versus lidocaine for median nerve block for intradermal procedures of the hand. The results indicated that the type and doses of medication had no significant relationship with onset time and duration of anesthesia induced by diphenhydramine, and both are comparable with what induced by lidocaine. Diphenhydramine injection, with any dose, was more painful than lidocaine injection. The mean pain score of two different used doses of diphenhydramine was not significant. As a result, patients receiving lidocaine had higher satisfaction level compared to patients receiving diphenhydramine. However, due to the absence of significant adverse effects, diphenhydramine can be considered as an appropriate alternative for lidocaine in median nerve block in patients with lidocaine hypersensitivity.

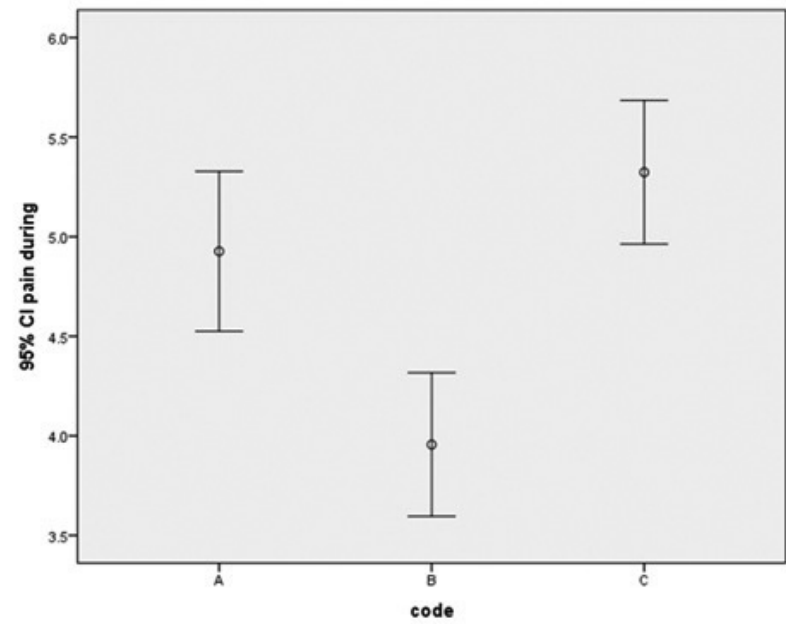

Figure 2. The pain score measured by Visual Analog Scale during injection of each group (A: 0.5\% Diphenhydramine Injection; B: 1\% Lidocaine Injection; C: 1\% Diphenhydramine Injection)

$\mathrm{Cl}$ : Confidence interval

Table 1. The quantitative and qualitative variables among treatment groups

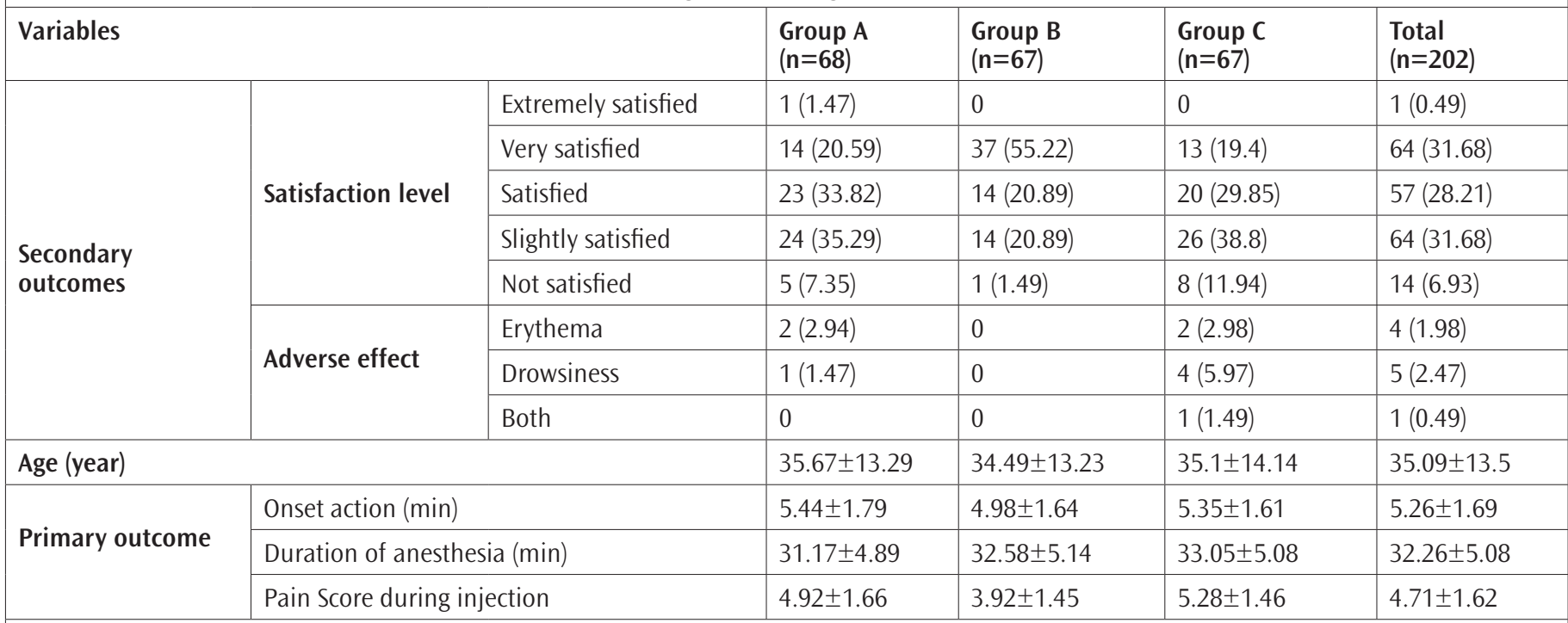

Date are frequency (percent) or mean \pm SD. A: 0.5\% Diphenhydramine Injection; B: 1\% Lidocaine Injection; C: 1\% Diphenhydramine Injection.

SD: Standard deviation, min: Minimum, n: Number 
In almost all previous studies, similar to the results of our study, it was reported that diphenhydramine is a suitable notquestionable alternative for lidocaine (4). Although there are some disagreements (16). Except rare ones (8), mostly pointed to the pain at the injection site as the main side effect of using diphenhydramine for local anesthesia, so tried to find a proper intervention to reduce it. To the best of our knowledge there is not any study, comparing two different doses of diphenhydramine in this regard. Various studies suggested that the technique of anesthetic injection is an important factor in the amount of pain during injection. Hence, appropriate training for using less painful techniques can reliably limit the pain of local anesthetic injection (17-19).

A study investigated the efficacy of $1.8 \mathrm{~mL}$ of $1 \%$ diphenhydramine with 1:100,000 epinephrine, $1.8 \mathrm{~mL}$ of $2 \%$ lidocaine with 1:100,000 epinephrine, and the combination of these two solutions in inferior alveolar nerve block. The results showed that post-injection discomfort using diphenhydramine was significantly higher than lidocaine. Although, the successful anesthesia for lidocaine was the most and for diphenhydramine was the least (5).

Twenty-four persons volunteered for intradermal injection of $0.5 \mathrm{~mL}$ diphenhydramine $1 \%$ and $2 \%$, lidocaine $1 \%$ and saline placebo. The efficacy of diphenhydramine $1 \%$ and lidocaine were significant higher than placebo and diphenhydramine $2 \%$. However, diphenhydramine $1 \%$ and lidocaine had priority with each other. No significant complication was reported. Generally, the pain score of diphenhydramine injection was higher (20).

In a study among 23 patients referred for oral surgery and needed local injection. Sixteen patients with positive history of allergy to local anesthesia received $2 \mathrm{~mL}$ diphenhydramine $1 \%$ and seven patients without allergy received $2 \mathrm{~mL}$ procaine $2 \%$. The results showed that the onset action of diphenhydramine was lower but the difference was no statistically significant. In addition, diphenhydramine had higher VAS score and lower duration of anesthesia. Using the diphenhydramine was accompanied with several complications including headache, bleeding, edema, pain and dizziness in one third of patients but no complication was observed in the use of procaine (21). When it comes to side effect, similar to our finding, different studies reported only limited and rare adverse effects of diphenhydramine and lidocaine. Hypersensitivity, pounding in chest and headache were some of adverse effects reported with lidocaine. Temporary hyperemia on injection site, erythema, tissue irritation, sedation and skin necrosis were some of the adverse effects of diphenhydramine $(6,7,22-25)$. Some studies claimed that the adverse effects of these two drugs can be reduced by decreasing concentration or volume of the medication, or by combining them with other drugs such as epinephrine $(4,7,26)$.

\section{Study Limitations}

In our study, the sample size was low due to the limited number of patients referred to ED with indication for median nerve block. Some variables such as age, gender, history of diabetes mellitus, hemodynamic changes, type and duration of procedure were not evaluated in the study. The incidence of adverse effects was very low which may be due to the excluding of all patients with history of hypersensitivity of diphenhydramine or lidocaine. In this regard, investigating the relationship between the adverse effects in different treatment groups was not statistically valuable. On the other hand, there was limited number of studies assessing the efficacy of diphenhydramine for median nerve block. Further studies are expected with higher sample size, different injection techniques and injection sites.

\section{Conclusion}

In summary, the present study evaluated the effect of two different concentrations of diphenhydramine and lidocaine in providing anesthesia via median nerve block, for simple hand procedures, and investigated the intensity of pain at injection site. The pain score of diphenhydramine injection, in its both concentrations, was higher than that of lidocaine, and patient satisfaction level was higher with lidocaine. Different doses of diphenhydramine did not result in a significant difference in onset of action or duration of anesthesia, or in pain score during injection.

\section{References}

1. Lovely LM, Chishti YZ, Woodland JL, Lalonde DH. How Much Volume of Local Anesthesia and How Long Should You Wait After Injection for an Effective Wrist Median Nerve Block? Hand (New York, NY). 2018;13:281-4.

2. Koay J, Orengo I. Application of local anesthetics in dermatologic surgery. Dermatol Surg. 2002;28:143-8.

3. Abdolrazaghnejad A, Banaie M, Tavakoli N, Safdari M, Rajabpour-Sanati A. Pain Management in the Emergency Department: a Review Article on Options and Methods. Adv J Emerg Med. 2018;2:e45

4. Bina B, Hersh EV, Hilario M, Alvarez K, McLaughlin B. True Allergy to Amide Local Anesthetics: A Review and Case Presentation. Anesth Prog. 2018;65:119-23.

5. Willett J, Reader A, Drum M, Nusstein J, Beck M. The anesthetic efficacy of diphenhydramine and the combination diphenhydramine/lidocaine for the inferior alveolar nerve block. J Endod. 2008;34:1446-50.

6. Pickett LE, Macdonald J. Injectable Diphenhydramine Used as Anaesthetic for Wide Local Excision With Flap Closure. J Cutan Med Surg. 2017;21:256-7.

7. Pavlidakey PG, Brodell EE, Helms SE. Diphenhydramine as an alternative local anesthetic agent. J Clin Aesthet Dermatol. 2009;2:37-40.

8. Xia Y, Chen E, Tibbits DL, Reilley TE, McSweeney TD. Comparison of effects of lidocaine hydrochloride, buffered lidocaine, diphenhydramine, and normal saline after intradermal injection. J Clin Anesth. 2002;14:339-43. 
9. Nakamura T, Popitz-Bergez F, Birknes J, Strichartz GR. The critical role of concentration for lidocaine block of peripheral nerve in vivo: studies of function and drug uptake in the rat. Anesthesiology. 2003;99:1189-97.

10. Liu J, Lv X. The pharmacokinetics and pharmacodynamics of lidocaineloaded biodegradable poly(lactic-co-glycolic acid) microspheres. Int J Mol Sci. 2014;15:17469-77.

11. Singer AJ, Hollander JE. Infiltration pain and local anesthetic effects of buffered vs plain 1\% diphenhydramine. Acad Emerg Med. 1995;2:884-8.

12. Delaunay L, Chelly JE. Blocks at the wrist provide effective anesthesia for carpal tunnel release. Can J Anaesth. 2001;48:656-60.

13. Faiz KW. [VAS--visual analog scale]. (Article in German).Tidsskrift for den Norske laegeforening : tidsskrift for praktisk medicin, ny raekke. 2014;134:323.

14. Arhami Dolatabadi A, Memary E, Kariman H, Nasiri Gigloo K, Baratloo A. Intranasal Desmopressin Compared with Intravenous Ketorolac for Pain Management of Patients with Renal Colic Referring to the Emergency Department: A Randomized Clinical Trial. Anesth Pain Med. 2017;7:e43595.

15. RANDOM.ORG website. What's this fuss about true randomness? [Available at: https://www.random.org/

16. Bartfield JM, Jandreau SW, Raccio-Robak N. Randomized Trial of Diphenhydramine Versus Benzyl Alcohol With Epinephrine as an Alternative to Lidocaine Local Anesthesia. Ann Emerg Med. 1998;32:650-4.

17. Strazar AR, Leynes PG, Lalonde DH. Minimizing the pain of local anesthesia injection. Plast Reconstr Surg. 2013;132:675-84.
18. Lalonde DH, Price C, Wong AL, Chokotho T. Minimally painful local anesthetic injection for cleft lip/nasal repair in grown patients. Plast Reconstr Surg Glob. 2014;2:e171-e.

19. Farhangkhoee H, Lalonde J, Lalonde DH. Teaching medical students and residents how to inject local anesthesia almost painlessly. Can J Plast Surg. 2012;20:169-72.

20. Green SM, Rothrock SG, Gorchynski J. Validation of Diphenhydramine as a Dermal Local Anesthetic. Ann Emerg Med. 1994;23:1284-9.

21. Uckan S, Guler N, Sumer M, Ungor M. Local anesthetic efficacy for oral surgery: Comparison of diphenhydramine and prilocaine. Oral Surg Oral Med Oral Pathol Oral Radiol Endod. 1998;86:26-30.

22. Tigka E, Alevizou A, Kotsiou A, Tesseromatis C. The Influence of Diphenhydramine Administration on Lidocaine Protein Binding in Rat Serum and Tissues. Pharmacology. 2011;7:880-3.

23. Lu DP. Managing patients with local anesthetic complications using alternative methods. Pa Dent J (Harrisb). 2002;69:22-9.

24. Boren E, Teuber SS, Naguwa SM, Gershwin ME. A critical review of local anesthetic sensitivity. Clin Rev Allergy Immunol. 2007;32:119-28.

25. Thyssen JP, Menne T, Elberling J, Plaschke P, Johansen JD. Hypersensitivity to local anaesthetics--update and proposal of evaluation algorithm. Contact Derm. 2008;59:69-78.

26. Klein JA, Jeske DR. Estimated Maximal Safe Dosages of Tumescent Lidocaine. Anesth Analg. 2016;122:1350-9. 